\title{
Contamination-free non-contact wettability assessment system
}

\author{
Nobuyuki Tanaka ${ }^{1 *}$, Yoshihide Haruzono ${ }^{2}$, Hiromitsu Nasu², Yuki Nakanishi', Junko Takahara' ${ }^{1}$ Akane Awazu ${ }^{1}$ \\ and Yo Tanaka**
}

\begin{abstract}
Surface wettability is one of most important indexes for understanding the interfacial characteristics between a solid surface and a liquid, especially such physico-chemical properties as hydrophilicity and water repellency. Recently, non-contact wettability assessment by monitoring the behavior of liquid covering a sample surface by jet-flow application to the liquid has been proposed. This method is applicable to the in-liquid assessment of high wettability materials for which it is difficult to measure the contact angle, such as cultured cells in a liquid culture medium. However, when the flow is dirty, the contamination of the cell culture system by particles (e.g. microorganisms, virus, and aerosols) may occur. To solve this problem, we developed a contamination-free non-contact wettability assessment system that is composed of a clean-air source having a particle filter and an automated system for image processing instrumentation and motorized alignment device for sample positioning. We confirmed that the filtered air-jet contained no particles, although over ten thousand particles had been included in the air source. Furthermore, we detected no microorganisms on agar culture plates, which had been exposed to the air-jet before being incubated. With the image instrumentation, we automatically measured the liquid-squeezed size, which was the main index of surface wettability. The motorized alignment device allowed us to control the sample position without human direct operation. This developed system is compatible with wettability assessment in a high-level clean environment such as quality control of culture cells for regenerative medicine and in vitro assay of drug efficacy and safety.
\end{abstract}

Keywords: Non-contact assessment, Wettability, Image instrumentation, Jet-flow, Contamination-free

\section{Background}

The surface wettability is an index of affinity between a liquid and a solid surface [1]. Because this index depends on the functional groups of surfaces, contaminants adhered on a surface, or surface roughness, the wettability value is useful for assessing physical and chemical properties. Especially, bio-surfaces such as biomaterials and cellular tissues are important targets for wettability assessment, because bio-surfaces interact with other materials via both physical and chemical interaction [21]. For example, temperature responsive cell culture dishes are important biomaterials for fabricating cell sheets based on the wettability

\footnotetext{
*Correspondence: nobuyuki.tanaka@riken.jp; yo.tanaka@riken.jp

${ }^{1}$ Quantitative Biology Center (QBiC), RIKEN. 1-3 Yamadaoka, Suita, Osaka 565-0871, Japan

Full list of author information is available at the end of the article
}

change on the dishes obtained by controlling the surface temperature $[4,13,23]$. Such cell sheet technologies are applicable to regenerative medicine for the malfunction of various organs and tissues $[2,3,5-7,11$, $12,15]$. Regarding cellular tissues, one possible application could be in treatment of dry eye syndrome; this is a corneal disorder wherein the corneal surface loses its tear film because of less secretion of tears and/or wettability loss on the corneal surface [10]. In analysis of disease occurrence, in vitro systems which use cell culture dishes are often used. Therefore, the wettability assessment method applicable to cell culture dish systems is in high demand.

The contact angle which is the angle on the contact interface between the liquid and solid is a popular index for expressing surface wettability of general materials such as metals, glasses, and resins [22]. However, measurement of the contact angle on high wettability surfaces 
such as hydrogels and cellular tissues is seldom done, because the liquid drop is immediately fused with the material surface. Furthermore, in the case of cellular tissues, the wet condition of tissues must be maintained, because drying on the surface causes cell damage. To keep the wet condition, a captive-bubble based contactangle measurement was developed [14], however, this measurement is difficult to apply to cell culture dish systems in vitro because the entire culture dish must be immersed into a liquid pool.

Recently, non-contact wettability assessment by monitoring the behavior of liquid covering a sample surface by air jet-flow application to the liquid has been proposed [18]. The area of liquid squeezed by the jet-flow application is the index of wettability in this method; small and large squeezed areas indicate high and low wettability, respectively (Fig. 1). This method is applicable to the in-liquid assessment of high wettability materials, such as cultivated oral mucosal epithelial cells $[8,9,17]$. In a previous study [19], we reported that the diameter of a culture medium, which initially covered the epithelial cells and then was squeezed by air-jet flow application, differed for culture conditions. Furthermore, higher wettability cells have been found to produce higher level of glycoproteins such as mucin. Although the method has been used to achieve wettability assessment for high wettability cellular surfaces in culture medium, jet-flow application presents a contamination risk for the cells. Therefore, our previous study only made a sampling assessment, and we were unable to cultivate the tested samples continuously and use them for further applications after the assessment. Because the contamination-free condition was not guaranteed, damages caused by the air-jet application and by particle contamination could not be distinguished separately. Furthermore, the usability of the non-contact wettability assessment method as compared to conventional methods such as contact angle measurement has not been quantitatively confirmed, though the basic relationship between liquid-squeezed size and contact angle is known (Fig. 1c-f).

There are two approaches to reduce the contamination risks: (1) improve the jet-flow cleanness and (2) lower the numbers of human operations, which introduce various contaminating particles. In this study, we incorporated the filtration of compressed air and robotic technologies such as position control and image processing to realize a contamination-free non-contact wettability assessment system using the above approaches. With the contamination-free system, we can evaluate the damage to cells from just the air-jet application and we can compare the cell wettability to the conventional wettability index, contact angle.
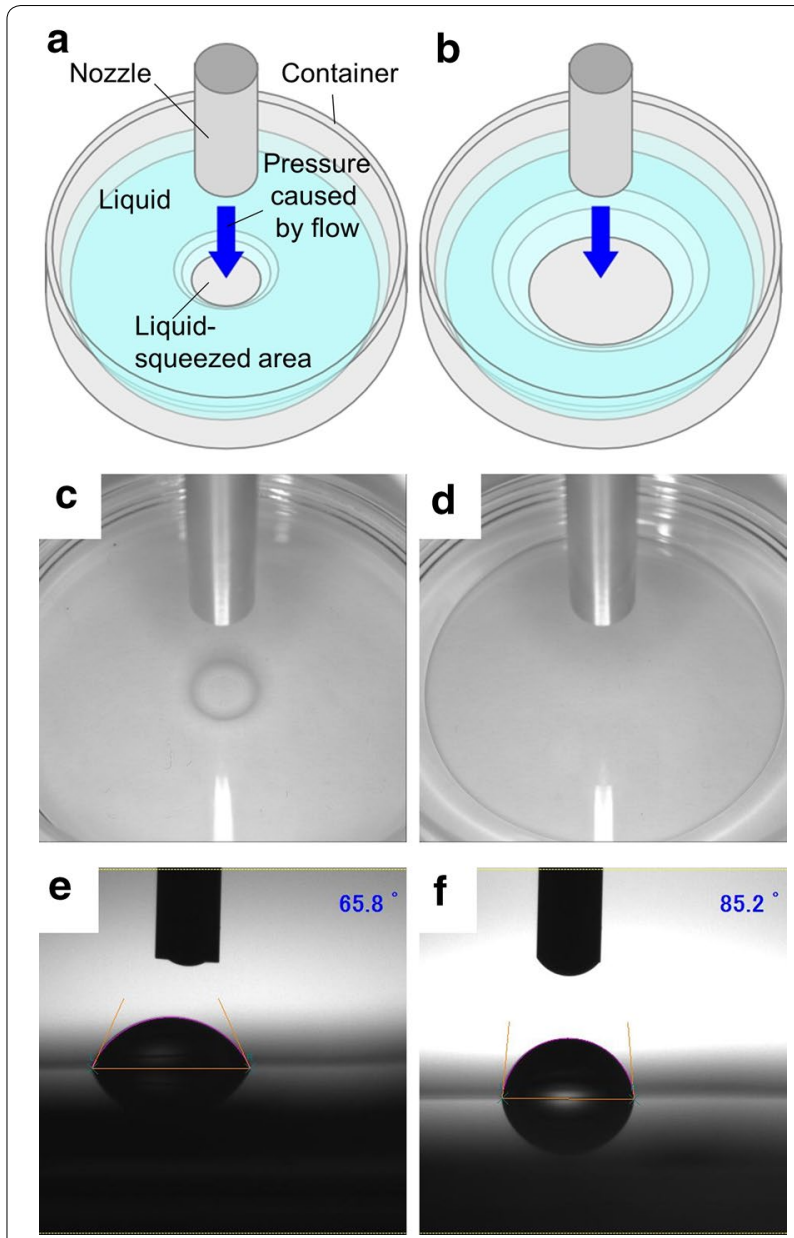

Fig. 1 Surface wettability assessment based on liquid squeezing by a jet flow. Schematic illustrations $\mathbf{a}, \mathbf{b}$ show the liquid squeezing on hydrophilic and hydrophobic surfaces, respectively. Photographs $\mathbf{c}, \mathbf{d}$ show the behaviors of ultra-pure water squeezed by an air-jet flow on hydrophilic and hydrophobic surfaces, corresponding to the contact angles of $65.8^{\circ}(\mathbf{e})$ and $85.2^{\circ}(\mathbf{f})$, respectively

\section{Results and discussion}

Non-contact wettability assessment system

We developed a non-contact wettability assessment system (Fig. 2a) based on our previous study [19] and related patent [20]. The system is equipped with a particle filter (Fig. 2a), an XYZ-three-axis motorized stage (Fig. 2b), and image processing instrumentation to allow detection of the liquid squeezed area automatically. The particle filter is readily available commercially as an item for clean rooms. Because various types of motorized stages are provided by several companies, we selected an appropriate type for the system from the viewpoints of position accuracy, size of the system and samples to be analyzed. The main system is W400 $\times \mathrm{L} 300 \times \mathrm{H} 250 \mathrm{~mm}$, and it can be installed on a standard clean bench. We also use a 


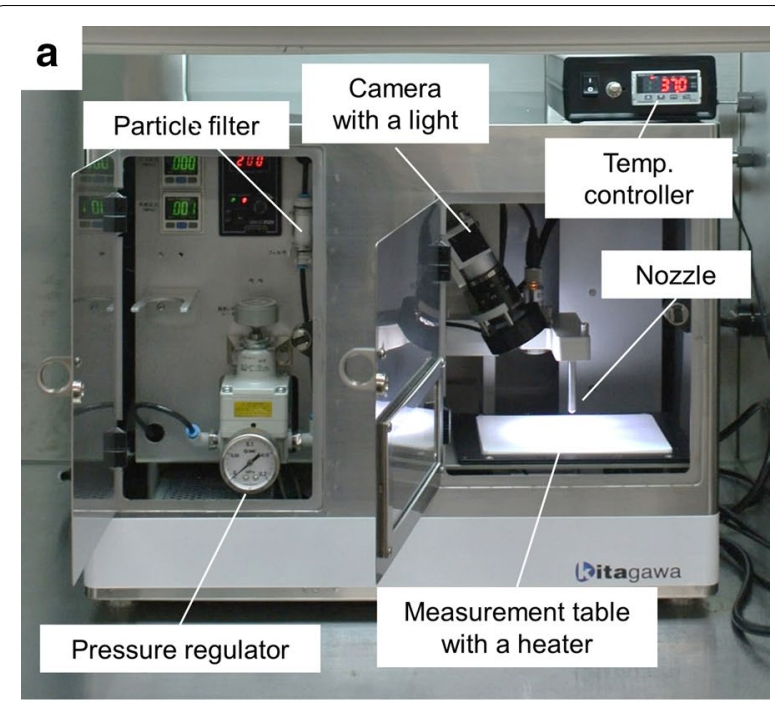

b

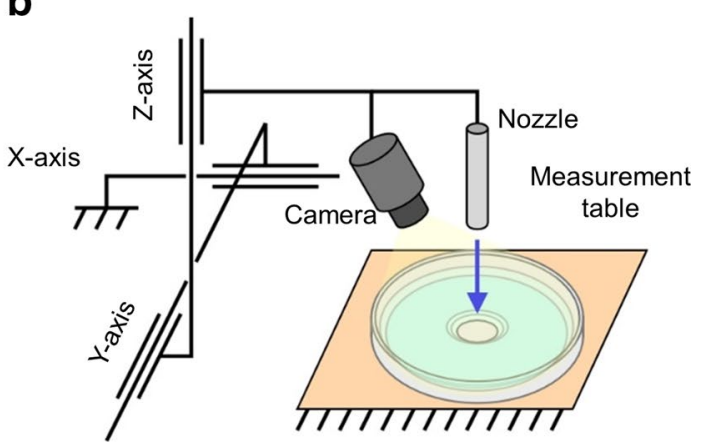

Fig. 2 Development of the non-contact wettability assessment system. a Photo of the system, consisting mainly of the jet-flow application and observation parts. b Schematic diagram showing the position adjustment mechanism

PC for system control and a compressed-gas source, such as an air compressor and a pressurized gas cylinder.

\section{Assessment of particle contamination}

First, we counted the particles in air flow from an oil-free air compressor (Fig. 3a) or non-contact wettability assessment system (Fig. 3b). The air flow from the compressor contained a large number of micro-particles: $1.8 \times 10^{4}$, $1.0 \times 10^{4}$, and $7.2 \times 10^{3}$ particles in a cubic foot with diameters of $0.3,0.5$, and $1.0 \mu \mathrm{m}$, respectively (Fig. 3c). These values were still smaller than that in a typical laboratory room, which reaches particle numbers of $10^{5}$ to $10^{6}$ particles (with a diameter of $0.3 \mu \mathrm{m}$ ) in a cubic foot. By installing the particle filter before the solenoid valve, the particles in the air-jet flow from the air nozzle were captured and removed (Fig. 3d). Therefore, filtration of the compressed air was quite useful for the risk reduction of particle contamination and necessary to achieve class
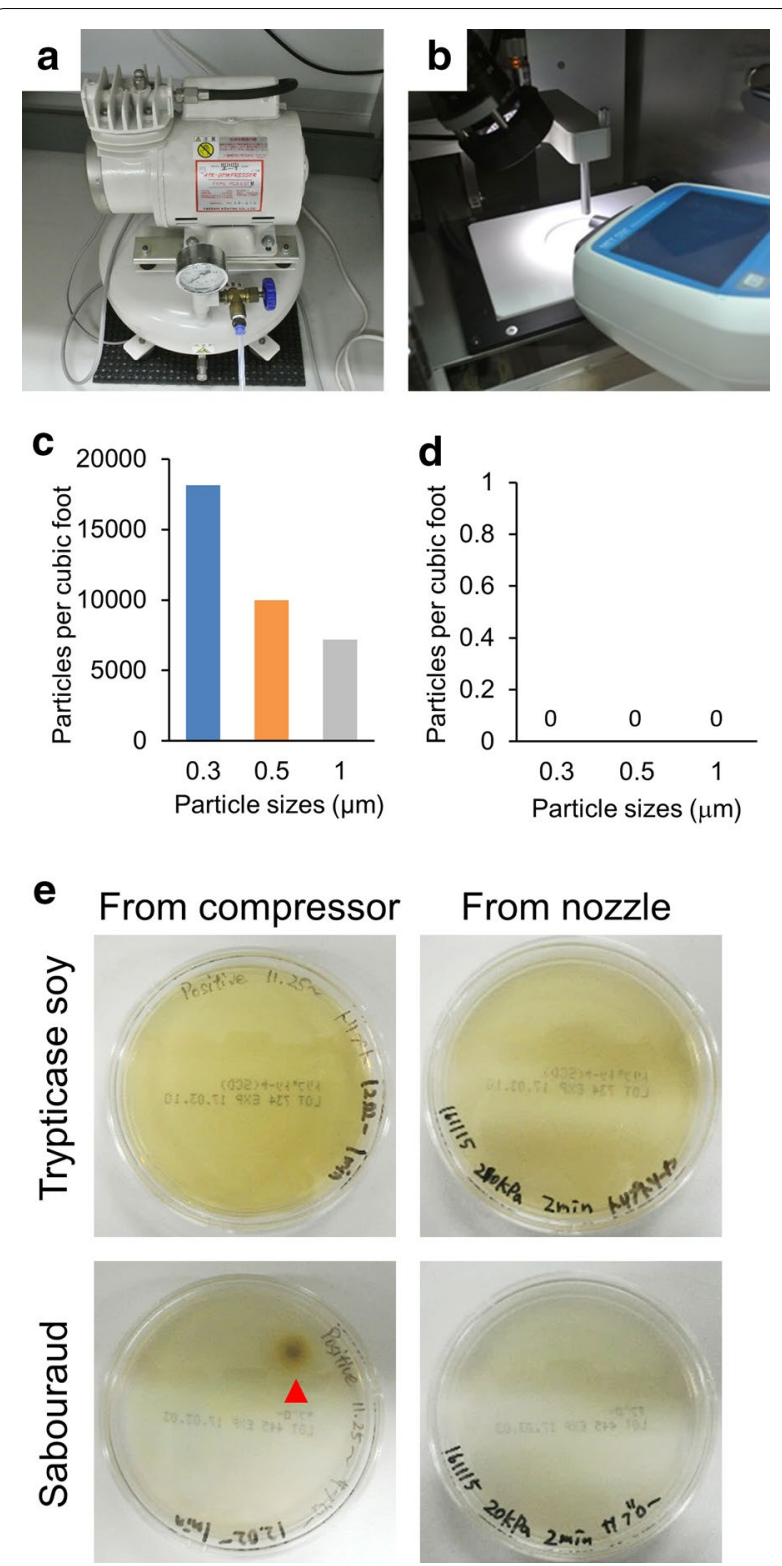

Fig. 3 Assessment of particle contamination risks. Two air-jet flows were investigated from: $\mathbf{a}$ an oil-free air compressor and $\mathbf{b}$ the air nozzle of the non-contact wettability assessment system. Graphs $\mathbf{c}$, d show the numbers of particles with the diameters of $0.3,0.5$, and $1 \mu \mathrm{m}$ in the two types of air-jet flows $(N=1)$. Photographs e show the results of micro-organism tests. The red arrow head indicates the colony of micro-organisms

5 level in the ISO 14644-1 cleanroom standards, which should be met to perform therapeutic cell culturing.

The microorganism test was also performed with agar culture plates (Fig. 3e). A colony was observed on the Sabouraud agar plate, which was exposed to the air directly from the air compressor and incubated. On the other hand, no colonies were observed on both trypticase 
soy and Sabouraud agar plates in the case of air-jet application from the air nozzle. Because trypticase soy and Sabouraud agar plates are suitable for cultivation of bacteria and fungi, respectively, the colony on the Sabouraud agar plate exposed to compressed air might consist of fungi derived from inside the air compressor. The result indicated that the air-jet from the non-contact wettability assessment system never contained bacteria and fungi. Therefore, the particles including microorganisms derived from the air compressor were effectively suppressed by insertion of the particle filter into the air supply line.

\section{Advantages obtained by using robotic technology and image processing instrumentation}

The main manual operation in the non-contact wettability assessment is the adjustment of measurement position, because the operator wants to make assessments on various points of the sample surface. The threeaxis motorized stage enables system users to avoid the manual handling of target samples. During continuous movement of the three-axis electric stage for $1 \mathrm{~min}$, no particles were detected. Although mechanical movement elements such as screws, bearings, and motors could be a particle source, the components of this system and the movement condition never generated particles.

To avoid the need for human operators to approach the measurement area, a digital camera and image processing instrumentation are included in the system. Obviously, these elements are not particle source, and the problem of contaminating particles derived from human operators was well eliminated. Furthermore, remote observations were implemented using software for the image instrumentation. This software detects the liquid squeezed area and measures its diameter. By automating this work, the time needed for analysis is shortened. In our previous study [19], human operators had to analyze huge numbers of recorded images to measure the diameter of the liquid squeezed area. But the simple algorithms of the image processing (Fig. 4a), allow the liquid squeezed area to be detected from just one monitored image (Fig. 4b-d; Additional file 3: Movie 1).

The performance of the image processing instrumentation was evaluated using circle patterns with known diameter values that had been printed on a sheet of paper (Fig. $4 \mathrm{e}-\mathrm{g}$ ). The diameter values of the actual and detected circles coincided within the worst coefficient of variation being $0.05 \%$ (=SD/mean) (Fig. $4 \mathrm{~h}$ ).

\section{Comparison with contact angle method}

To confirm the usability of our developed system, we compared the two indexes, liquid-squeezed diameter during air-jet application and the liquid recovery time after ceasing the air-jet application, with the conventional contact angle measurement on the same surfaces (Fig. 5). To obtain surfaces with various wettability values, polystyrene petri dishes were treated with atmospheric pressure nitrogen-plasma for $0,3,6,12,18,30$, and $60 \mathrm{~s}$. With the extension of treatment time, the contact angle was decreased, indicating that the wettability was improved (Fig. 5a). Both liquid-squeezed diameter and liquid recovery time were also measured on these

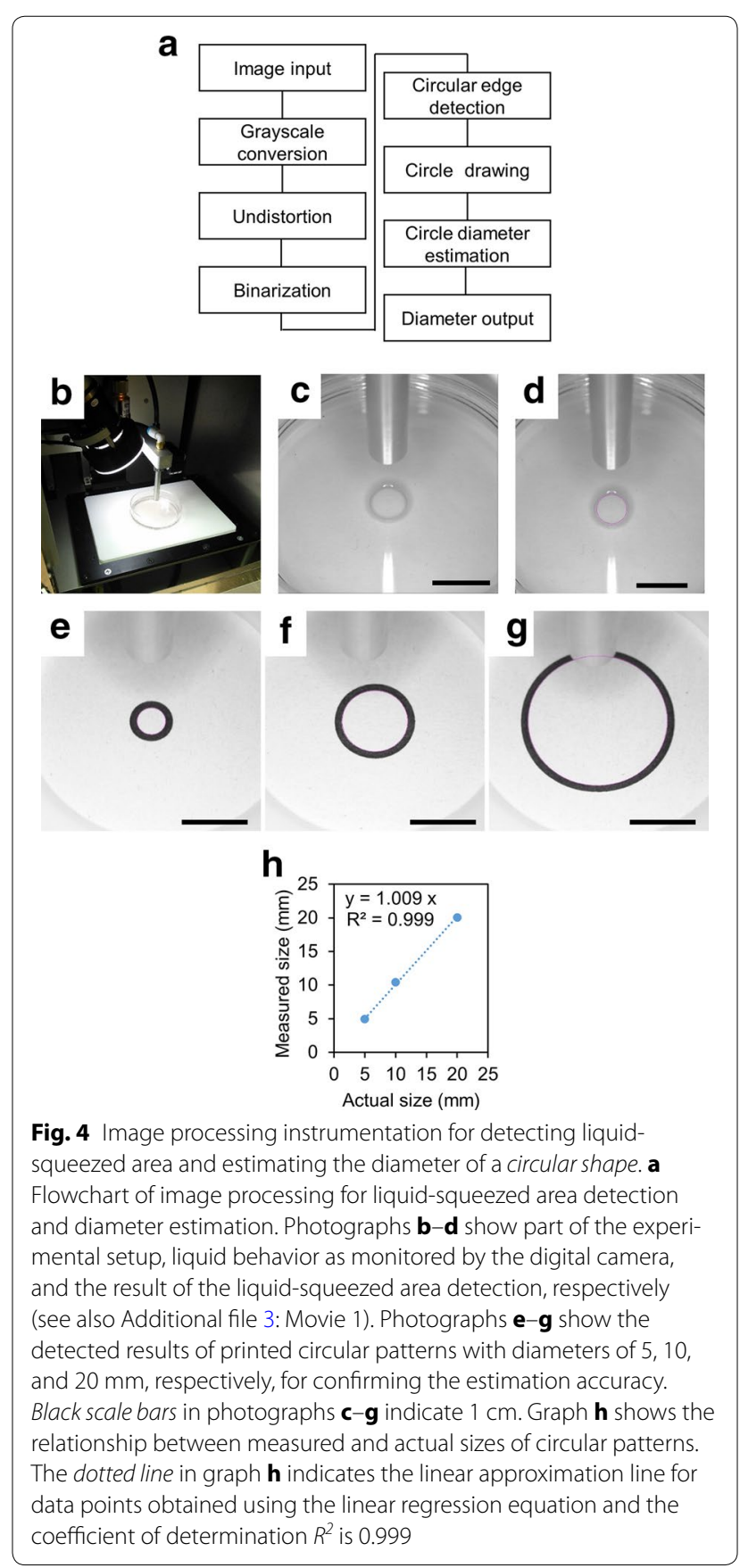



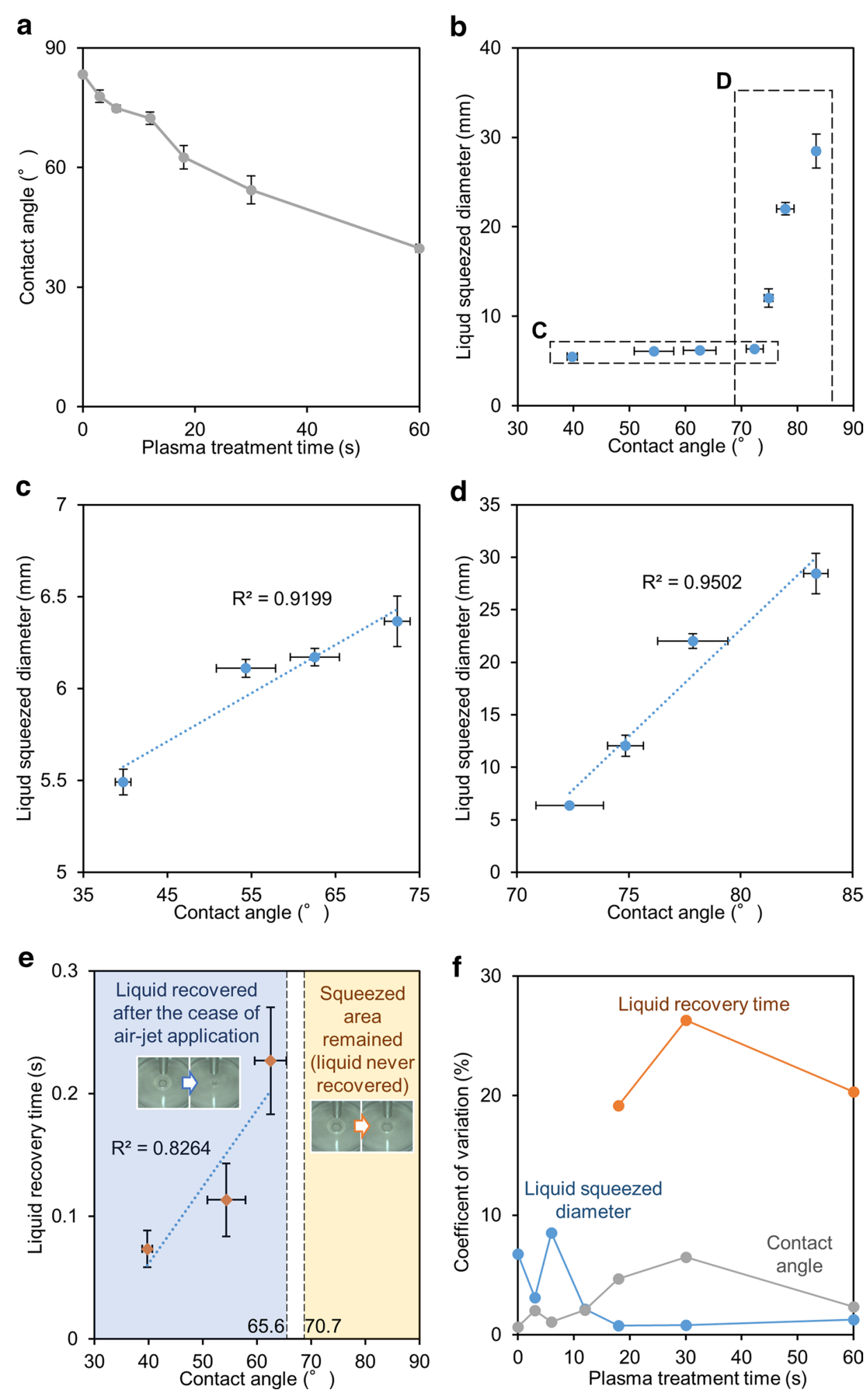


\section{(See figure on previous page.)}

Fig. 5 Comparison between a conventional contact angle method and our air-jet based liquid squeezing method as realized in the non-contact wettability assessment system. Graph a shows the relationship between contact angle on the polystyrene surfaces treated with atmospheric-pressure nitrogen-plasma and this treatment time. Graphs $\mathbf{b}-\mathbf{d}$ show the relationship between liquid squeezing diameter during air-jet application and contact angle. Dashed-line rectangles $\mathbf{c}, \mathbf{d}$ in graph $\mathbf{b}$ indicate the region shown in graphs $\mathbf{c}, \mathbf{d}$, respectively. Graph $\mathbf{e}$ shows the relationship between liquid recovery time after ceasing the air-jet application and contact angle on the polystyrene surfaces. The blue-and yellow-background regions in graph e indicate recovering of liquid and the remaining squeezed area after ceasing the air-jet application, respectively, as shown in the paired photographs in graph $\mathbf{e}$. The dashed vertical lines in graph $\mathbf{e}$ indicate the boundaries between the liquid recovery and the remaining squeezed area in the experiment and the values at the bottom end of each line are the contact angles at the boundaries. Dotted lines in graphs $\mathbf{c}-\mathbf{e}$ are linear approximation lines for data points ( $R^{2}$ the coefficient of determination is shown in each graph). Data points and error bars indicate mean values and standard deviations ( $n=5$ ), respectively. Graph $\mathbf{f}$ shows the relationship between the coefficient of variation (=standard deviation/mean value) and plasma treatment time for contact angle, liquid-squeezed diameter, and liquid recovery time

surfaces with the developed system and the relationship to the contact angle was investigated. The line expressing the relationship between the liquid-squeezed diameter and contact angle had a positive slope (Fig. 5b) with a small squeezed diameter at a small contact angle and a large diameter at a large angle, as observed earlier in Fig. 1c-f. Interestingly, the increase of the liquidsqueezed diameter had two different slopes with the turning point being around $72^{\circ}$ for the contact angle; that is, the slope for the contact angle below $72^{\circ}$ (Fig. 5c) was quite a bit gentler than that over $72^{\circ}$ (Fig. 5d). In each region (Fig. 5c, d), the relationship seemed to be almost linear and the linear approximation lines well fit the data points with a coefficient of determination $R^{2}$ over 0.9 . Unfortunately, we could not identify the mechanism causing the slope change; however, we could confirm assessment usability between the liquid-squeezed diameter and contact angle, which is the gold standard among wettability indexes, but contact angle measurement cannot be applied to cell surfaces.

Through this experiment, we investigated the relationship between the liquid recovery dynamics after ceasing the air-jet application and the contact angle (Fig. 5e). In the case of the surface with a contact angle over $70.7^{\circ}$, the liquid never recovered the surface and the liquid squeezed area caused by the air-jet application remained. On the other hand, the surface with a contact angle below $65.6^{\circ}$ was always recovered by the liquid. Furthermore, whenever the liquid recovery occurred, the liquid recovery time increased with the contact angle. And this result suggested that the liquid recovery time could be used as an index of wettability, especially for high wettability surfaces with small values of the contact angle. Supposing the relationship was modelled as linear, we saw the approximation line provided a good estimation of contact angle from the liquid recovery time with the coefficient of determination $R^{2}$ over 0.8 .

The coefficient of variation $(\mathrm{CV})$, which was defined as the standard deviation per mean value, of each wettability index was calculated (Fig. 5f). On a low wettability surface with plasma treatment time from 0 to $12 \mathrm{~s}$, the contact angle was the best index among the three indexes. On the other hand, the $\mathrm{CV}$ value of liquid-squeezed diameter was the best index for a higher wettability surface with 18-s or more treatment time. Therefore, the airjet based liquid squeezing method implemented in our non-contact wettability assessment system would be the most suitable for the in-liquid wettability assessment of high wettability surfaces. The liquid recovery time had 4-30 times larger CV values compared with those of contact angle and liquid-squeezed diameter. Because the liquid recovery is a quite fast behavior, the measurement system with more frequent sampling, which exceeds the current system with a normal video rate of $30 \mathrm{~Hz}$, is required for accurate evaluation.

We found the contact angle measured in the gas phase was strongly dependent on ambient humidity and the trend for the dependency varied with the surface materials (Additional file 1: Figure S1). Therefore, the ambient humidity must be controlled for accurate measurement of contact angle. On the other hand, this non-contact wettability assessment system is free from the effect of ambient humidity, because it is an in-liquid method, although the proposed method cannot be applicable to the wettability assessment in dry condition.

\section{Influence on cultivated cells during wettability assessment} To investigate physical and biochemical influences during wettability assessment, we cultivated mouse skeletal myoblast cells on cell culture dishes (Fig. 6a).

First, mechanical strength was assessed by observing peeling off of cultivated cells from the dish during air-jet application. However, cells were never peeled from the dish and the cellular shape was seemingly never changed before and after the air-jet application of $10 \mathrm{kPa}$ pressure (Fig. 6b, c). When a gentler air-jet is required to reduce the pressure on the surface, not only the regulation of gas pressure but also the nozzle height from the surface is effective. From the spatial distribution of pressure (Additional file 2: Figure S2), the maximum pressure, which 

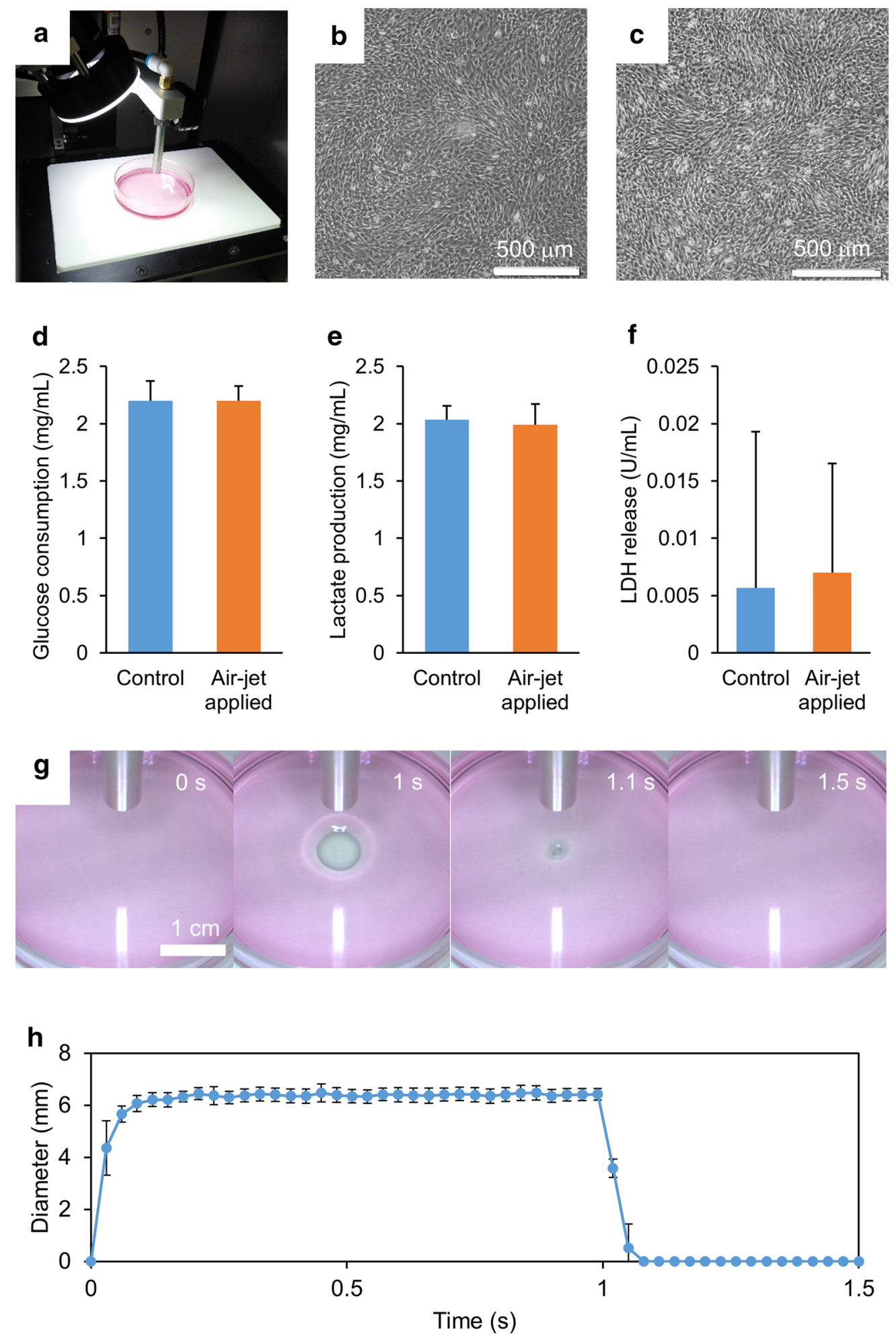

Fig. 6 Influence on cultivated cells during non-contact wettability assessment. a Photo showing part of the experimental set-up. Microphotographs $\mathbf{b}$, c show the surfaces of cultivated cells before and after 10-kPa-pressure air-jet application, respectively. Graphs $\mathbf{d}-\mathbf{f}$ show the glucose consumption, lactate production, and lactate dehydrogenase release of the culture system during a 1-day culturing after noncontact wettability assessment, respectively. Bars and error bars indicate average values and standard deviations $(n=3)$, respectively. $\mathbf{g}$ Series of monitored images showing culture medium during non-contact wettability assessment (see also Additional file 4: Movie 2). Graph $\mathbf{h}$ shows the time-course of the liquid-squeezed diameter. Data points and error bars indicate mean values and standard deviations $(n=3)$, respectively 
was caused at the point just under the nozzle center, can be suppressed by controlling the nozzle height.

Next, to investigate biochemical influences, glycometabolism and damage to the cytomembrane were assessed for the $10 \mathrm{kPa}$ air-jet application, which never caused the peeling off of cells. The condition without the air-jet application was defined as the control. Generally, cells consume glucose and convert it into lactate in glycometabolism. Therefore, the concentration changes of both glucose and lactate in culture media were quantified during a 1-day culturing after the air-jet application (Fig. 6d, e). As a result, we saw no differences in the glucose consumptions and lactate productions between the control and air-jet application conditions. The released concentrations of lactate dehydrogenase (LDH) were also quantified to assess damage to the cytomembrane, because LDH is normally located in the cytoplasm and released through a damaged cytomembrane (Fig. 6f). The LDH release from cells showed no significant difference between the control and air-jet application conditions. Therefore, these results suggested that there were no influences on the cells during the wettability assessment in comparison with the control condition.

\section{Wettability assessment}

Here, we assessed the wettability of cultivated mouse skeletal myoblasts with air-jet based liquid squeezing. The behavior of the liquid squeezing and liquid recovery could be monitored by the developed system (Fig. 6g; Additional file 2) and we analyzed the image data to obtain the time-courses of squeezed diameter from the image instrumentation (Fig. 6h). The time-courses of three individual measurements were in almost complete agreement. As compared with the squeezed diameter using the normal polystyrene dish during air-jet application (Fig. 4b; Additional file 3: Movie 1), the diameter of the squeezed culture medium area was almost the same as that when using the dish. On the other hand, although the squeezed area remained in the case of the polystyrene dish after the air-jet cut-off, the culture medium fully recovered the cell culture surface (Additional file 3: Movie 1, Additional file 4: Movie 2). Therefore, these results suggested that hysteresis between liquid-squeezing and rewetting was also important information about surface wetting.

\section{Conclusions}

In this study, we developed a contamination-free noncontact wettability assessment system by installing robotic technology and image processing instrumentation for applications in biomedical fields. Air-jet application at a $10 \mathrm{kPa}$ pressure never caused peeling off of cells and it did not influence biochemical activities including glycometabolism and it did not cause cytomembrane damage. Because the micro-particles including microorganisms can be eliminated by air filtration, the system will be applicable to in vitro assessment of wettability on cell surfaces during culturing.

\section{Methods}

\section{System setup}

The non-contact wettability assessment system was mainly composed of two parts: (1) an air-jet application part and (2) a part for the acquisition and analysis of liquid behavior. The air-jet application part had a compressed air line from an air compressor as an air source (PC3-5.5T; Yaezaki Kuatsu, Tokyo, Japan) to an air nozzle and the line was equipped with a pressure regulator (IR2000-A; SMC Corporation, Tokyo, Japan), a particle filter (ZFC53; SMC), and solenoid valve (custom-made; $\mathrm{SMC}$ ). Both pressures before and after the solenoid valve were monitored by pressure sensors (PSE540; SMC). The air-jet was switched by the solenoid valve that was controlled from a laptop computer (ProBook650G1; HP, Palo Alto, CA). The liquid behavior in a sample container on the measurement table of the system was monitored by a digital camera located above the table. The surface temperature of the measurement table was controlled by a heater according to the user's requirements. The relative position between the air nozzle and measurement table could be adjusted with the XYZ-three-axis motorized stage (XY-axes: KYL06, Suruga Seiki, Shizuoka, Japan and Z-axis: DRSM42, Oriental Motor, Tokyo, Japan) via numerical control from the computer. The acquired images were transferred to the computer and then further analyzed by our in-house software.

\section{Particle counting}

All experiments were performed with a particle counter (MET ONE HHPC 3+; Beckman Coulter, Brea, CA) in a laminar flow type clean bench (MCV-710ATS; Panasonic Healthcare, Tokyo, Japan). Before the air supply was fed from the air sources, we confirmed no particles could be counted in the clean bench. For the air compressor (PC3-5.5T; Yaezaki Kuatsu, Tokyo, Japan), the air was introduced into the particle counter in the clean bench via a tube. When the air-jet from the air nozzle of the non-contact wettability assessment system was assessed, first, the system was installed into the clean bench and put into the stand-by mode until no particles were detected in the bench. Then, the air-jet was applied to the particle counter (Fig. 3b). The three different sizes of particles in the air were simultaneously counted by the particle counter. 


\section{Microorganism test}

Two types of agar plates were used for the microorganism test: trypticase soy (51048; Nissui Pharmaceutical, Tokyo, Japan) and Sabouraud agar plates (51033; Nissui). Each agar plate was exposed to the air from air compressor or the air nozzle of our system for $1 \mathrm{~min}$. After being covered with a lid, the plates were incubated at $37^{\circ} \mathrm{C}$ for 2 weeks. After the incubation, the colonies were monitored.

\section{Contact angle measurement}

A contact angle meter (DMs-401; Kyowa Interface Science, Saitama, Japan) were used for assessing the wettability of the surface as a standard method. A $10 \mu \mathrm{L}$ droplet of ultra-pure water was applied to the surface to be evaluated. The contact behavior between the droplet and surface was monitored with a digital camera equipped in the contact angle meter. The value of the contact angle was automatically measured from the camera image using the software of the contact angle meter. To obtain surfaces with various levels of wettability, polystyrene petri dishes (351007, Corning, Corning, NY) and vacuum-gas-plasma (VGP) treated polystyrene cell culture dishes (353002; Corning) were prepared. The polystyrene petri dishes were further treated with nitrogen-plasma generated by a pen-style atmospheric-pressure plasma device (P500-SM; Sakigake Semiconductor, Kyoto, Japan).

\section{Cell culturing}

The cell culturing of mouse skeletal myoblast cell line C2C12 was performed as described elsewhere [16]. Briefly, Dulbecco's modified Eagle's Medium (DMEM) (A6429; Sigma-Aldrich, St. Louis, MO) supplemented with $10 \mathrm{v} / \mathrm{v} \%$ fetal bovine serum (FBS) (Nichirei Biosciences, Tokyo, Japan) and 1v/v\% penicillin-streptomycin solution (168-23191; Wako Pure Chemical, Osaka, Japan) was prepared as a culture medium. Cells were seeded in the culture medium in a $60-\mathrm{mm}$ cell culture dish (353002; Corning) at an initial density of $6 \times 10^{4}$ cells $/ \mathrm{cm}^{2}$. Cells were cultivated for 5 days in a humidified condition with $5 \% \mathrm{CO}_{2}$ at $37{ }^{\circ} \mathrm{C}$. Cell morphology was monitored with a phase-contrast microscope (IX71; Olympus, Tokyo, Japan).

\section{Wettability assessment}

For assessing the wettability of $60-\mathrm{mm}$ polystyrene petri and cell culture dishes to an ultra-pure water (electrical resistivity: $\geq 18 \mathrm{M} \Omega \mathrm{cm}$ ), $2.7 \mathrm{~mL}$ of ultra-pure water was poured into the dish. In case of the assessment for cultivated cells, the same volume of culture medium was poured in cell culture dish covered with the cells at confluence. The volume of liquid $(2.7 \mathrm{~mL})$ gave a liquid height of $1.2 \mathrm{~mm}$ in the dish. After the target dish was placed on the measurement table of the system, the airjet was applied from the air nozzle at the height of $15 \mathrm{~mm}$ from the liquid surface with a regulated pressure of $10 \mathrm{kPa}$. The behavior of liquid was recorded by the system camera from $0.1 \mathrm{~s}$ before the $1 \mathrm{~s}$ air-jet application to $0.5 \mathrm{~s}$ after ceasing it.

\section{Image instrumentation}

Digital camera images showing liquid behavior were transferred from the digital camera embedded in the non-contact wettability assessment system to a laptop computer. The images were analyzed by image processing software for detecting the liquid-squeezed area based on a circle detection algorithm as described in the flowchart of Fig. 4a. In the case of an absence of liquidsqueezed area, the software provided a diameter of zero as an undetected signal. To determine the diameter of the liquid-squeezed area, the relationship between actual distance and pixel distance in the image was calibrated in advance.

\section{Pressure distribution measurement}

The spatial distribution of pressure caused by the airjet application was measured with a digital manometer (8230; AZ Instrument, Taichung, Taiwan). The air-jet at the regulated pressure of $10 \mathrm{kPa}$ was applied to the pressure sensor located under the air nozzle of the developed system. The relative position of the pressure sensor to the nozzle was changed as heights of 5,10 , and $15 \mathrm{~mm}$ and distances from the point just under the nozzle between 0 to $5 \mathrm{~mm}$ in $1-\mathrm{mm}$ increments.

\section{Additional files}

Additional file 1: Figure S1. Comparison of measured contact angle in different humidity environments. Photographs (A) show the $10-\mu \mathrm{L}$-volume droplet on the bare and vacuum-gas-plasma (VGP) treated surfaces of polystyrene in five different environments humidified at 39,51,61, 71, and $80 \%$ relative humidity $(\mathrm{RH})$. Graph $(\mathrm{B})$ shows the relationship between the value of the contact angle and \% $\mathrm{RH}$. Data points and error bars indicate mean values and standard deviations ( $n=5)$, respectively.

Additional file 2: Figure S2. Pressure distribution caused by the air-jet application with different nozzle heights of 5,10, and $15 \mathrm{~mm}$. Data points and error bars indicate mean values and standard deviations $(n=6)$, respectively.

Additional file 3: Movie 1. Squeezing ultra-pure water covering a polystyrene dish by 10-kPa-pressure air-jet and the result of squeezed area detection

Additional file 4: Movie 2. Squeezing cell culture medium covering cultivated mouse skeletal myoblasts on a cell culture dish by 10-kPa-pressure air-jet and the result of squeezed area detection.

\section{Abbreviations}

QBiC: Quantitative Biology Center; LDH: lactate dehydrogenase; DMEM: Dulbecco's modified Eagle's Medium; FBS: fetal bovine serum; KIW: Kitagawa Iron Works. 


\section{Authors' contributions}

$\mathrm{NT}$ and $\mathrm{YH}$ initiated the project. $\mathrm{YH}$ and $\mathrm{HN}$ designed and developed the system. NT designed the experiments and wrote the paper. NT, YN, JT, and AA performed the experiments and analyzed data. YT supervised the project. All authors read and approved the final manuscript.

\section{Author details}

${ }^{1}$ Quantitative Biology Center (QBiC), RIKEN. 1-3 Yamadaoka, Suita, Osaka 565-0871, Japan. ${ }^{2}$ Development Division, Kitagawa Iron Works, 77-1 Motomachi, Fuchu-shi, Hiroshima 726-8610, Japan.

\section{Competing interests}

The complimentary prototype of the non-contact wettability assessment system was provided from Kitagawa Iron Works (KIW) to RIKEN under the collaborative research agreement between KIW and RIKEN.

\section{Availability of data and materials}

Four Additional files 1, 2, 3 and 4 are available.

\section{Consent for publication}

Not applicable.

Ethics approval and consent to participate Not applicable.

\section{Funding}

The study was partially supported by Adaptable and Seamless Technology Transfer Program through Target-driven R\&D (AS2715165U) from the Japan Science and Technology Agency.

\section{Publisher's Note}

Springer Nature remains neutral with regard to jurisdictional claims in published maps and institutional affiliations.

Received: 27 April 2017 Accepted: 11 August 2017

Published online: 19 September 2017

\section{References}

1. Bhattacharya S, Datta A, Berg JM, Gangopadhyay S (2005) Studies on surface wettability of poly(dimethyl) siloxane (PDMS) and glass under oxygen-plasma treatment and correlation with bond strength. J Microelectromech Syst 14:590-597. doi:10.1109/JMEMS.2005.844746

2. Burillon C, Huot L, Justin V, Nataf S (2016) (CAOMECS) transplantation for the treatment of corneal limbal epithelial stem. Cell Deficiency. doi:10.1167/iovs.11-7744

3. Ebihara G, Sato M, Yamato M et al (2012) Cartilage repair in transplanted scaffold-free chondrocyte sheets using a minipig model. Biomaterials 33:3846-3851. doi:10.1016/j.biomaterials.2012.01.056

4. Egami M, Haraguchi Y, Shimizu T et al (2014) Latest status of the clinical and industrial applications of cell sheet engineering and regenerative medicine. Arch Pharm Res 37:96-106. doi:10.1007/s12272-013-0299-8

5. Iwata T, Washio K, Yoshida T et al (2015) Cell sheet engineering and its application for periodontal regeneration. J. Tissue Eng Regen Med 9:343-356

6. Iwata T, Yamato M, Tsuchioka H et al (2009) Periodontal regeneration with multi-layered periodontal ligament-derived cell sheets in a canine model. Biomaterials 30:2716-2723. doi:10.1016/j.biomaterials.2009.01.032

7. Kaneshiro N, Sato M, Ishihara M et al (2006) Bioengineered chondrocyte sheets may be potentially useful for the treatment of partial thickness defects of articular cartilage. Biochem Biophys Res Commun 349:723731. doi:10.1016/j.bbrc.2006.08.096

8. Kondo M, Yamato M, Takagi R et al (2013) The regulation of epithelial cell proliferation and growth by $\mathrm{LL}-1$ receptor antagonist. Biomaterials 34:121-129. doi:10.1016/j.biomaterials.2012.09.036

9. Kondo M, Yamato M, Takagi R et al (2014) Significantly different proliferative potential of oral mucosal epithelial cells between six animal species. J Biomed Mater Res, Part A 102:1829-1837. doi:10.1002/jbm.a.34849
10. Lemp BYMA, Foulks GN (2008) The definition \& classification of dry eye disease. Ocul Surf 5:75-92

11. Nishida K, Yamato M, Hayashida Y et al (2004) Corneal reconstruction with tissue-engineered cell sheets composed of autologous oral mucosal epithelium. N Engl J Med 351:1187-1196. doi:10.1056/NEJMoa040455

12. Ohki T, Yamato M, Ota M et al (2012) Prevention of esophageal stricture after endoscopic submucosal dissection using tissue-engineered cell sheets. Gastroenterology 143:582-588. doi:10.1053/j.gastro.2012.04.050

13. Okano T, Yamada N, Sakai H, Sakurai Y (1993) A novel recovery system for cultured cells using plasma-treated polystyrene dishes grafted with poly(N-isopropylacrylamide). J Biomed Mater Res 27:1243-1251. doi:10.1002/jbm.820271005

14. Pettit DK, Horbett TA, Hoffman AS, Chan KY (1990) Quantitation of rabbit corneal epithelial cell outgrowth on polymeric substrates in vitro. Invest Ophthalmol Vis Sci 31:2269-2277

15. Sawa Y, Miyagawa S, Sakaguchi T et al (2012) Tissue engineered myoblast sheets improved cardiac function sufficiently to discontinue LVAS in a patient with DCM: report of a case. Surg Today 42:181-184. doi:10.1007/ s00595-011-0106-4

16. Tadakuma K, Tanaka N, Haraguchi Y et al (2013) A device for the rapid transfer/transplantation of living cell sheets with the absence of cell damage. Biomaterials 34:9018-9025. doi:10.1016/j.biomaterials.2013.08.006

17. Takagi R, Yamato M, Murakami D et al (2011) Fabrication and validation of autologous human oral mucosal epithelial cell sheets to prevent stenosis after esophageal endoscopic submucosal dissection. Pathobiology 78:311-319. doi:10.1159/000322575

18. Tanaka N, Kaneko M, Uchida R et al (2012) Noncontact evaluation of the wetting characteristic of a cell sheet in culture medium. IEEE Int Conf Mechatronics Autom 2012:986-991. doi:10.1109/ICMA.2012.6283384

19. Tanaka N, Kondo M, Uchida R et al (2013) Splitting culture medium by air-jet and rewetting for the assessment of the wettability of cultured epithelial cell surfaces. Biomaterials 34:9082-9088. doi:10.1016/j. biomaterials.2013.08.029

20. Tanaka N, Uchida R, Kondo M, et al (2013b) Object wettability evaluation method. PCT/JP2013/064510, 28 Nov 2013.

21. Ueda-Yukoshi T, Matsuda T (1995) Cellular responses on a wettability gradient surface with continuous variations in surface compositions of carbonate and hydroxyl groups. Langmuir 11:4135-4140. doi:10.1021/ la00010a080

22. Wenzel RN (1936) Resistance of solid surfaces to wetting by water. Ind Eng Chem 28:988-994. doi:10.1021/ie50320a024

23. Yamada N, Okano T, Sakai H et al (1990) Thermo-responsive polymeric surfaces; control of attachment and detachment of cultured cells. Die Makromol Chemie Rapid Commun 11:571-576. doi:10.1002/ marc.1990.030111109

\section{Submit your manuscript to a SpringerOpen ${ }^{\circ}$ journal and benefit from:}

- Convenient online submission

$\checkmark$ Rigorous peer review

- Open access: articles freely available online

- High visibility within the field

- Retaining the copyright to your article

Submit your next manuscript at springeropen.com 